Artículos Científicos

\title{
Analfabetismo hídrico en el municipio de Xalisco, Nayarit: EI reto para mejorar la gobernanza del agua y la seguridad hídrica
}

Water Illiteracy in the Municipality of Xalisco, Nayarit: The Challenge to Improve Water Governance and Water Security

Analfabetismo hídrico no município de Xalisco, Nayarit: o desafio de melhorar a governança da água e a segurança hídrica

Nicté Rosas Topete Universidad Autónoma de Nayarit, México nicte.uan@gmail.com https://orcid.org/0000-0002-6009-1835

Edgar Gabriel Ávila Verdín Universidad Autónoma de Nayarit, México edgaravilav1@hotmail.com https://orcid.org/0000-0001-6004-0029

Isma Sandoval Galaviz Universidad Autónoma de Nayarit, México ismasangal@yahoo.com.mx https://orcid.org/0000-0002-6533-5130 


\section{Revista Iberoamericana \\ de las Ciencias Sociales y \\ Humanísticas}

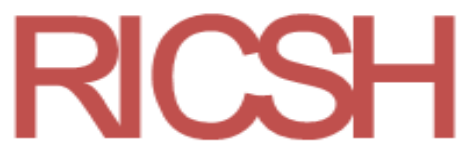

ISSN: $2395-7972$

\section{Resumen}

El valor del agua tiene un limitado reconocimiento en México. El poco conocimiento de los ciudadanos acerca de la disponibilidad del recurso ha propiciado desperdicio, sobreexplotación y deterioro de la calidad del agua. El objetivo de este estudio fue visibilizar el nivel del analfabetismo hídrico en el municipio de Xalisco, Nayarit. Se recurrió a un enfoque mixto, diseño explicativo y nivel aplicativo; y se aplicó una encuesta a una población basada en una muestra no probabilística de 1000 personas de la cabecera municipal. Dicha encuesta constó de 65 preguntas distribuidas en cinco dimensiones de análisis. El criterio de aplicación se basó en ocho rutas, cubriendo la totalidad de la zona geográfica, la cual se conforma por 29 colonias. El análisis estadístico se realizó con el software Dyane versión 4, mediante tabulaciones simples.

La mayoría de la población encuestada fueron mujeres (54.9\%). Del total, $96.8 \%$ tiene conexión de agua potable. Respecto de los habitantes por domicilio, de uno a tres representan 50.4 $\%$ y de cuatro a seis $46 \%$, lo que indica un alto porcentaje de habitantes por hogar. De la visibilidad de la problemática, las respuestas indican que desde $57.1 \%$ hasta $91.3 \%$ de la población reconoce en diferentes aspectos la situación que guarda el recurso hídrico. Entre las actividades en las que se utiliza el recurso con mayor frecuencia se encuentran las relacionadas al aseo personal (80.6\%) y las relacionadas con el uso doméstico, las cuales se encuentran entre porcentajes de $52.2 \%$ hasta $56.8 \%$. De los servicios, $52.8 \%$ desconoce cuál es el organismo que lo provee; $84.8 \%$ señala que el servicio es de bueno a regular. Un dato relevante es la falta de pago por el servicio, representada por $6.8 \%$, del cual $3.8 \%$ señala como causa la falta de consecuencias. Sobre el cuidado y conservación, 40.7 \% señala la desinformación como razón para no cuidar el agua, $39 \%$ no se da cuenta que la desperdicia y $33.8 \%$ por desinterés. Además, $47.5 \%$ desconoce algún método para reutilizar el agua y $63.9 \%$ no lo hace; la sumatoria de los niveles medio, poco y nulo de responsabilidad para cuidarla representa $77.7 \%$. Asimismo, $49.7 \%$ considera que la población en general es la principal responsable de cuidarla, mientras que $30.7 \%$ señala a los usuarios, organismo operador y Gobierno. Del conocimiento y participación, 61 \% no conoce información suficiente para cuidar y preservar el agua, $81.4 \%$ consideran importantes las campañas informativas y $77 \%$ estaría dispuesto a participar en actividades para cuidarla. Por último, más de $80 \%$ desconoce las leyes relacionadas con el recurso hídrico, $75.5 \%$ considera importante que existan, $86.8 \%$ que la autoridad las aplique y $88.9 \%$ que los usuarios las cumplan. 


\section{Revista Iberoamericana \\ de las Ciencias Sociales y \\ Humanísticas}

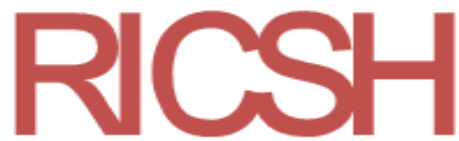

ISSN: $2395-7972$

En los resultados obtenidos se observa un alto índice de analfabetismo hídrico en la población. Sin embargo, se encontraron aspectos de disposición en la ciudadanía para su disminución a través de una participación más activa e involucramiento en actividades para el cuidado, preservación y una verdadera cultura del agua. En suma, se reconoce la importancia de la problemática y la necesidad de realizar acciones conjuntas entre la sociedad y el Gobierno.

Palabras clave: conservación del agua, derecho del agua, educación no formal, gestión de los recursos hídricos, participación social, política gubernamental.

\section{Abstract}

The value of water has a limited recognition in Mexico. The little information that citizens know about the availability of the resource has led to inefficient use, waste, overexploitation and deterioration of water quality. The objective of this study was to make visible the impact of water illiteracy in the municipality of Xalisco, Nayarit. A mixed approach, explanatory design and application level was used; and a survey was applied to a population based on a non-probabilistic sample of 1000 people from the municipal capital. The above mentioned survey consisted of 65 questions distributed in five dimensions of analysis. The application criterion was based on eight routes, covering the entire geographical area, which is made up of 29 colonias. The statistical analysis was performed with the DYANE V4 software through simple tabulations.

The majority of the surveyed population were women (54.9\%). Of the total, $96.8 \%$ have a drinking water connection. Regarding the inhabitants by domicile, from one to three they represent $50.4 \%$ and from four to six $46 \%$, which indicates a high percentage of inhabitants by domicile. About the visibility of the problem, the answers indicate that from $57.1 \%$ to $91.3 \%$ of the population recognizes in different aspects the situation related to water. Among the activities that use the resource most frequently are those related to personal hygiene $80.6 \%$ and those related to domestic use, which are between $52.2 \%$ to $56.8 \%$. Related to the services, $52.8 \%$ do not know which is the agency that provides it, $84.8 \%$ indicate that the service is good or regulate. A relevant fact is the lack of payment for the service, represented by $6.8 \%$, of which $3.8 \%$ indicate as cause the lack of consequences. About the care and conservation, $40.7 \%$ indicate misinformation as a reason for not taking care of water, 39\% do not realize that they waste it and 33.8 because of lack of interest. Further, $47.5 \%$ do not know any method to reuse water, and $63.9 \%$ do not reuse; the sum of the medium, little and no responsibility levels to care it represents $77.7 \%$. Also, $49.7 \%$ believes that the general population is the main responsible for caring, whereas $30.7 \%$ indicates 


\section{Revista Iberoamericana \\ de las Ciencias Sociales y \\ Humanísticas}

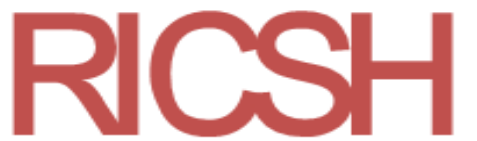

ISSN: $2395-7972$

the users, organization and government. About knowledge and participation, we found that $61 \%$ do not know enough information to care for and preserve water, $81.4 \%$ consider information campaigns important, and $77 \%$ would be willing to participate in activities to care for it. Finally, more than $80 \%$ do not know the laws related to water resources, $75.5 \%$ consider important that they exist, $86.8 \%$ that the authority applies them, and $88.9 \%$ that users comply with them.

The results obtained show a high rate of water illiteracy in the population. However, aspects of disposition of the citizens for its reduction were found, through a more active participation and involvement in activities for the care, preservation and, a true water culture, recognizing the importance of the problem and the needs for joint actions of society and government.

Keywords: water conservation, water law, nonformal education, water resources management, social participation, government policy.

\section{Resumo}

O valor da água tem um reconhecimento limitado no México. O pouco conhecimento dos cidadãos sobre a disponibilidade do recurso levou ao desperdício, à superexploração e à deterioração da qualidade da água. O objetivo deste estudo foi tornar visível o nível de analfabetismo hídrico no município de Xalisco, Nayarit. Uma abordagem mista, design explicativo e nível de aplicação foram utilizados; e uma pesquisa foi aplicada a uma população baseada em uma amostra não probabilística de 1000 pessoas da sede municipal. Este levantamento consistiu em 65 questões distribuídas em cinco dimensões de análise. O critério de aplicação foi baseado em oito rotas, cobrindo toda a área geográfica, composta por 29 colônias. A análise estatística foi realizada com o software Dyane versão 4, utilizando tabulações simples.

A maioria da população pesquisada era do sexo feminino (54,9\%). Do total, 96,8\% possuem conexão com água potável. Em relação aos habitantes por domicílio, de um a três eles representam $50,4 \%$ e de quatro a seis $46 \%$, o que indica um alto percentual de habitantes por domicílio. A partir da visibilidade do problema, as respostas indicam que de $57,1 \%$ a $91,3 \%$ da população reconhece em diferentes aspectos a situação que o recurso hídrico comporta. Entre as atividades em que o recurso é mais utilizado estão as relacionadas à higiene pessoal $(80,6 \%)$ e aquelas relacionadas ao uso doméstico, que estão entre 52,2\% a 56,8\%. Dos serviços, 52,8\% não sabem qual é a agência que o fornece; $84,8 \%$ indicam que o serviço é bom para regular. Fato relevante é a falta de pagamento pelo serviço, representada por $6,8 \%$, dos quais $3,8 \%$ indicam como causa a falta de 


\section{Revista Iberoamericana \\ de las Ciencias Sociales y \\ Humanísticas}

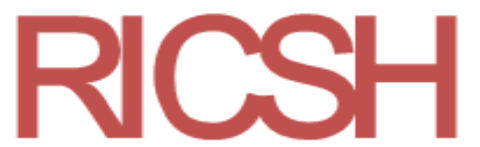

ISSN: 2395 - 7972

conseqüências. Sobre o cuidado e a conservação, 40,7\% indicam desinformação como motivo para não cuidar da água, 39\% não percebem que é desperdiçado e 33,8\% por falta de interesse. Além disso, 47,5\% não conhecem nenhum método de reutilização de água e 63,9\% não o utilizam; a soma da média, pouco e nenhum nível de responsabilidade para cuidar dela representa 77,7\%. Da mesma forma, 49,7\% consideram que a população geral é a principal responsável por cuidar dela, enquanto 30,7\% indicam os usuários, a agência operacional e o governo. De conhecimento e participação, $61 \%$ não conhecem informações suficientes para cuidar e preservar a água, 81,4\% consideram importantes as campanhas de informação e $77 \%$ estariam dispostas a participar de atividades para cuidar dela. Por fim, mais de $80 \%$ não conhecem as leis relacionadas aos recursos hídricos, 75,5\% consideram importante que existam, 86,8\% que a autoridade as aplica e 88,9\% que os usuários as cumprem.

Os resultados obtidos mostram um alto índice de analfabetismo hídrico na população. No entanto, aspectos da disposição dos cidadãos para diminuir foram encontrados através de uma participação mais ativa e envolvimento em atividades de cuidado, preservação e uma verdadeira cultura da água. Em suma, reconhece a importância do problema e a necessidade de realizar ações conjuntas entre a sociedade e o governo.

Palavras-chave: conservação da água, legislação sobre a água, educação não formal, gestão dos recursos hídricos, participação social, política governamental.

Fecha Recepción: Noviembre 2018

Fecha Aceptación: Junio 2019

\section{Introducción}

¿Dónde, cómo y cuándo surge la problemática? Durante mucho tiempo se pensó que los recursos naturales, entre ellos el agua, eran inagotables y que siempre estarían disponibles para la satisfacción de nuestras necesidades; aún hoy, existen muchas personas que piensan que se puede acceder a ellos como cosas que no tienen dueño y que nadie tendría que preocuparse por su cuidado y renovabilidad, por la creencia de que son autorenovables. Las preocupaciones en torno a los recursos naturales se centraban en utilizarlos para promover el crecimiento económico y el mejoramiento de la calidad de vida. A inicios del siglo XXI, surge una crisis mundial de agua que impacta de manera sustancial la seguridad, la estabilidad y el equilibrio del medio ambiente. Por tanto, la problemática ambiental de los tiempos actuales nos ha enfrentado con un panorama distinto, muchos recursos naturales están en grave peligro por la sobreexplotación, y en lo que concierne al agua, la ilegalidad en su uso, la ocupación indebida de los cauces y las zonas de 


\section{Revista Iberoamericana \\ de las Ciencias Sociales y \\ Humanísticas}

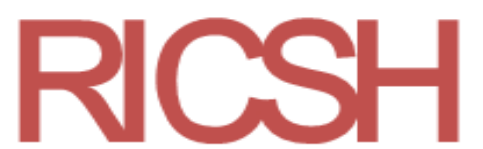

ISSN: $2395-7972$

protección, los conflictos por su uso y la contaminación cada vez más incontrolada del recurso hídrico, ponen en riesgo la disponibilidad de este líquido vital en términos de cantidad y calidad (Organización de las Naciones Unidas para la Educación, la Ciencia y la Cultura [Unesco], 2018).

El valor del agua tiene un limitado reconocimiento en nuestro país. El conocimiento que los ciudadanos tienen acerca de la disponibilidad del recurso ha propiciado el uso ineficiente, desperdicio, sobreexplotación y deterioro de la calidad del agua. Por lo anterior, la educación y cultura son elementos sustanciales para la transformación de actitudes, valores y conductas para su protección, conservación y preservación (Comisión Nacional del Agua [Conagua], 2013). Ante el panorama descrito, surge el término analfabetismo hídrico, que podemos interpretar como el desconocimiento o falta de información relacionada con el agua que abastece a las localidades, así como la falta de conocimiento sobre quiénes son responsables de la administración, esto es, elementos sustanciales para facilitar la comprensión de la importancia del vital líquido y contribuir a su aprovechamiento de una forma sustentable.

El estado de Nayarit está incluido en dos regiones hidrológicas administrativas: la III Pacífico Norte y la VII Lerma Santiago Pacífico. La primera cuenta con una disponibilidad natural media de $25681 \mathrm{hm}^{3} /$ año y la segunda con $34003 \mathrm{hm} 3 /$ año. Su precipitación pluvial anual es de $1061.6 \mathrm{~mm}$, que contrasta con los $175.6 \mathrm{~mm}$ de Baja California Sur y los 2424.1 de Tabasco (Comisión Nacional del Agua [Conagua], 2013). La condición de Nayarit por la disponibilidad de agua es privilegiada en el conjunto de las entidades federativas. Es el estado de la región CentroOccidente con mayor disponibilidad de agua. Sin embargo, adolece de serias deficiencias en su gestión; área en la que se ha generado un rezago importante.

Al respecto, $95 \%$ de las comunidades del estado, principalmente en áreas rurales, no cuentan con el vital líquido, aun cuando $91 \%$ del total de viviendas tiene agua entubada, lo que habla de que los principales rezagos se observan en las áreas marginadas principalmente de las zonas serranas, donde la topografía y la lejanía de las comunidades hace muy difícil la disposición de agua.

Ahora bien, de acuerdo con los datos del Sistema Nacional de Información Municipal [SNIM] publicados en 2010, el municipio de Xalisco cuenta con 37 localidades. Tiene una superficie de $504 \mathrm{~km}^{2}$ y representa $1.79 \%$ de la superficie del estado de Nayarit. La cabecera municipal Xalisco tiene 35702 habitantes, de los cuales 17363 son hombres y 18339 mujeres. Las coordenadas geográficas de la cabecera municipal son las siguientes: $21^{\circ} 26^{\prime} 39^{\prime \prime} \mathrm{N}, 1^{\circ} 4^{\circ} 54^{\prime}$ 06" O, y con una altitud de $995 \mathrm{msnm}$. En el municipio, al igual que en el estado, se han identificado 


\section{Revista Iberoamericana \\ de las Ciencias Sociales y \\ Humanísticas}

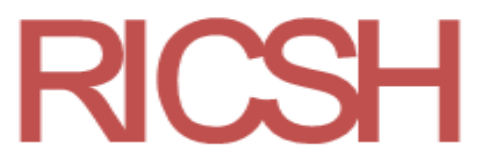

ISSN: $2395-7972$

como principales problemas la falta de recursos en organismos operadores; falta de mantenimiento de la infraestructura hidráulica; insuficiencia operativa de los organismos operadores; obsolescencia de los sistemas hidráulicos; elevados costos de la infraestructura hidráulica; falta de capacitación del personal operativo; falta de una cultura del agua y de su uso eficiente, así como la existencia de una cultura de no pago del servicio. Al ser el agua un factor esencial en el desarrollo y un firme soporte para reducir las desigualdades, la existencia, disponibilidad y gestión del agua resultan ser elementos de primer orden para garantizar el progreso y el avance de las sociedades.

El problema del agua, uno de los acuciantes del presente siglo, puesto de manifiesto en el seno del IV Foro Mundial del Agua en Marsella, Francia, en 2012, es esencialmente un problema de gobernabilidad y gobernanza, por tanto, un problema político en primera instancia; no es solamente un conflicto de escasez del elemento o del financiamiento para su disponibilidad, puesto que para una adecuada administración del recurso son necesarias instituciones sólidas, esfuerzos de cooperación y capacidad de decisión en todos los niveles (Organización para la Cooperación y el Desarrollo Económicos [OCDE], 2012). El principal reto a considerar, de acuerdo con lo anterior, radica en la implementación de soluciones que partan de la especificidad de las localidades, con amplia participación de los actores involucrados, mecanismos de rendición de cuentas y la aceptación de riesgos y responsabilidades para conseguir resultados en el marco de la equidad y de la sustentabilidad del recurso y su saneamiento. De ahí que más que una política generalizable a todo el Estado, se tenga la necesidad de diseñar políticas municipales contextualizadas que tengan en cuenta lo específico de sus territorios y el involucramiento de la sociedad.

La Asociación Mundial para el Agua (GWP, por su siglas en inglés) define la gobernabilidad del agua como "el conjunto de sistemas políticos, sociales, económicos y administrativos establecidos para desarrollar y gestionar los recursos hídricos y la prestación de servicios de agua en diferentes estratos de la sociedad" (OCDE, 2012, p. 29). Por tanto, es necesario fortalecer la gobernabilidad de los recursos hídricos a través de procesos democráticos, es decir, considerando la gobernanza (OCDE, 2012). En este propósito, la gobernanza se refiere a una serie de cambios que se suscitan entre el Gobierno y la sociedad, que reconstruyen el sentido, capacidad y dirección en relación con el medio ambiente. Asimismo, como una mayor capacidad de decisión e influencia de los actores no gubernamentales en la definición, creación e implementación de políticas públicas o la prestación de servicios relacionados con el agua. También, se refiere a los 


\section{Revista Iberoamericana \\ de las Ciencias Sociales y \\ Humanísticas}

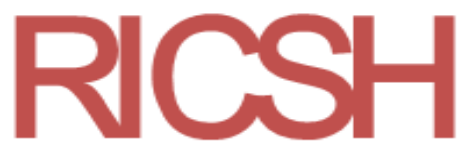

ISSN: $2395-7972$

procesos y mecanismos de interacción entre los actores gubernamentales y no gubernamentales. Se trata, pues, de un concepto dinámico.

La gobernanza se concibe como un proceso y no como un fin. Se plantean acercamientos flexibles para adaptarse a las circunstancias, y se abordan temas prioritarios relativos tanto al recurso en sí mismo (contaminación, escasez, deterioro, sobreexplotación) como a la gestión (ausencia de legislación, escasos recursos, información, etcétera). Aunado a lo anterior, se concibe como un proceso reactivo frente a la ausencia de voluntad política para realizar cambios sustanciales que beneficien a la sociedad (Organización de los Estados Americanos [OEA], 2017). La administración pública, desde esta perspectiva, debe ser incluyente, democrática y responsable, considerando a los ciudadanos no solo como contribuyentes, sino que además como consumidores, clientes, evaluadores y electores; derivado de lo anterior, se asumirán como responsables y solidarios, incluso, participando activamente en los procesos relacionados con el recurso (Rosas, Campos y Calderon, 2012).

En este sentido, la gobernabilidad del agua en México en los últimos 30 años se ha llevado a cabo mediante la implantación de dos modelos diferentes (Musetta, 2009). El primero de ellos caracterizado por un Estado fuerte, que planificó y buscó el desarrollo mediante la construcción de grandes obras de infraestructura hidráulica, tal y como diques, represas, sistemas de riego, al mismo tiempo que organizó a los demás actores de la economía y de la sociedad. En este periodo (19601990), el Estado funge como legítima autoridad sobre el agua sin que se conciba un actor distinto. El segundo modelo, el de la gobernanza, surge como consecuencia de las reformas neoliberales de los 90 y obedece a un cambio en cuanto a la concepción del Estado como rector y organizador del orden general: un Estado que empieza a compartir este papel con otros actores; y se desarrollan diversas formas de intervención estatal, pero no necesariamente de un retiro del Estado y de su poder, de tal forma que en la actualidad coexisten los dos modelos. Así, pues, es uno de los mecanismos que consolidan y en donde se desarrollan los supuestos del modelo de gobernanza, el de la participación social.

Esta participación está contemplada en la Ley de Aguas Nacionales y permite el diseño de un nuevo sistema de negociación con los actores económicos, privados y de la sociedad civil que son convocados a formar parte de dicho modelo de gestión del agua, con la promoción por parte del Estado. Así, la misión de la Conagua, máximo organismo a nivel nacional que tiene que ver con el recurso hídrico, se centra en administrar y preservar las aguas nacionales con la participación de la sociedad con vistas a lograr el uso sustentable del agua. Sin embargo, la implantación de este 


\section{Revista Iberoamericana \\ de las Ciencias Sociales y \\ Humanísticas}

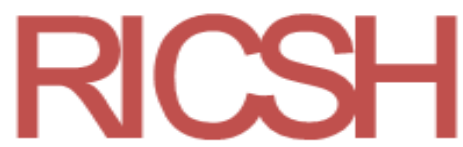

ISSN: 2395 - 7972

modelo mixto no ha estado exenta de dificultades (Domínguez, 2013): se han detectado problemas en lo que respecta a la superposición de programas gubernamentales del orden federal con los estatales, la falta de coordinación o la falta de infraestructura, personal o recursos destinados al agua; falta de comunicación intramunicipal, asignación de tarifas deficientes, ausencia de capacidades de gestión ambiental, entre otras.

Los Objetivos de Desarrollo Sostenible (ODS) que se adoptaron y fueron promovidos por la Organización de las Naciones Unidas (ONU) en el año 2015 originaron una ocasión única para que los países impulsaran una variedad de temas críticos, especialmente en materia ambiental. Particularmente, la propuesta actual del sexto objetivo, a saber: "Garantizar la disponibilidad de agua y su gestión sostenible y el saneamiento para todos" (Programa de las Naciones Unidas para el Desarrollo [PNUD], 2016).

Por lo anterior, se considera que la seguridad hídrica es un factor fundamental para afirmar y mantener, además de mejorar, la calidad de vida de los seres humanos. Se enfatiza en realizar acciones conjuntas en los campos de la política, la educación, la investigación y la práctica. Además, pretende que se mejore el conocimiento de la cantidad, calidad y el uso del recurso hídrico. Y para ello aboga por desarrollar mecanismos que permitan prever, controlar, eficientar el uso del recurso y establecer un parámetro para que la demanda corresponda con la disponibilidad; aunado a mejorar la gobernanza del agua (Jimenez, 2015).

En contraste con el crecimiento económico obtenido en las recientes décadas, somos testigos de la agudización de muchos problemas sociales entre los cuales destacan los relacionados con la disponibilidad y aprovechamiento del agua. Si bien es cierto que la infraestructura, utilización racional del recurso y la conformación de organismos técnicos de distribución del mismo siguen siendo de extraordinaria importancia para garantizar el acceso a este bien de forma sustentable y con criterios de género, el recurso humano es un elemento de gran valor para garantizar el acceso al vital líquido. Por lo anterior, el presente estudio pretende visibilizar el nivel del analfabetismo hídrico en el municipio de Xalisco,Nayarit,

A partir de lo expuesto se plantea que mientras menos esté informada la población sobre el uso, cuidado y preservación del recurso hídrico, más se limita la posibilidad de establecer rutas de acción para el rediseño, regulación, implementación y evaluación eficaz de los mecanismos existentes a efectos de propiciar mejoras que permitan implementar un modelo de gobernanza adecuado a las necesidades de la sociedad. 


\section{Revista Iberoamericana \\ de las Ciencias Sociales y \\ Humanísticas}

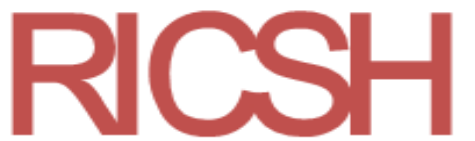

ISSN: $2395-7972$

\section{Método}

Para el desarrollo del estudio y organización de los conocimientos se utilizaron los métodos sistemático, inductivo, deductivo, analítico, sintético y científico, además de las écnicas documental y de investigación de campo, puesto que, por la complejidad del problema a enfrentar, demanda ser observado desde varias dimensiones. Se espera que sirva como base para la formulación y aplicación de mejoras en las políticas públicas tendientes a fortalecer el acceso a la información, participación ciudadana y aplicación de las leyes existentes, y propicie con ello el uso del recurso con criterios de equidad, eficiencia, eficacia y sustentabilidad. La categoría central de este trabajo fue el analfabetismo hídrico en la población de la cabecera municipal de Xalisco, Nayarit.

Se utilizó un enfoque mixto, un diseño explicativo y se trabajó en el nivel aplicativo. Se aplicó una encuesta a una población basada en una muestra no probabilística. Lo anterior con base en la distribución geográfica del municipio y por ser un diagnóstico inicial de la problemática. Por tanto, se conformó de 1000 personas de la cabecera municipal. Cabe señalar que la encuesta constó de 65 preguntas distribuidas en cinco dimensiones de análisis; el criterio de aplicación se basó en ocho rutas (véase figura 1), con lo que se cubrió la totalidad de la zona geográfica, la cual se conforma por 29 colonias.

Las dimensiones en las que se distribuyeron los cuestionamientos son las siguientes:

- D0. Información general.

- $\quad$ D1. Visibilidad de la problemática del agua.

- D2. Servicios.

- D3. Cuidado y conservación.

- D4. Conocimiento y Participación.

- D5. Marco Legal. 


\section{Revista Iberoamericana \\ de las Ciencias Sociales y \\ Humanísticas}

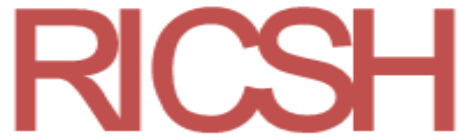

ISSN: $2395-7972$

Figura 1. Distribución de rutas para aplicación en la zona geográfica

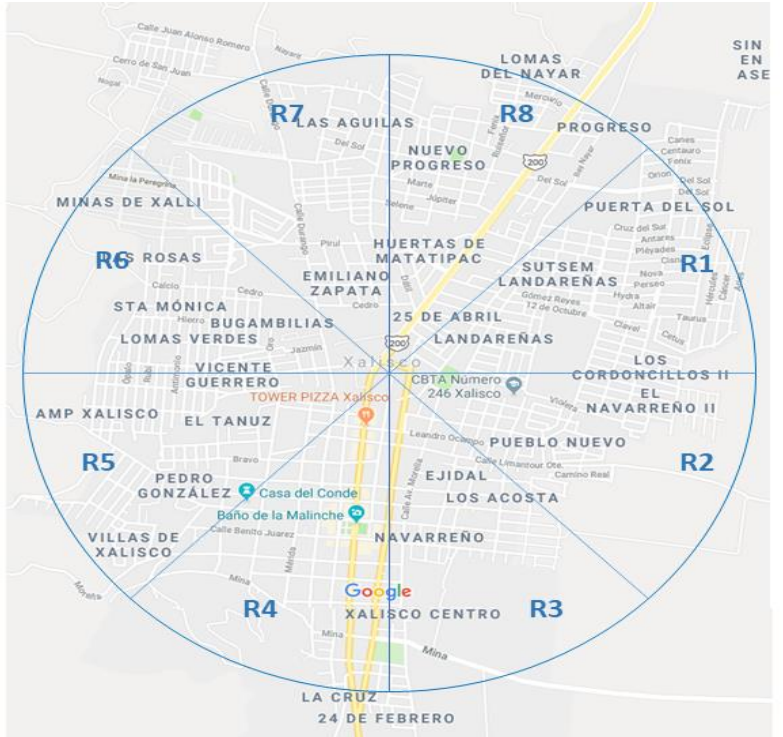

Fuente: Elaboración propia con imagen de Google Maps

El análisis estadístico se realizó con el software Dyane versión 4 y se realizaron tabulaciones simples. Asimismo, se estableció un consentimiento informado a efectos de garantizar los aspectos éticos del estudio, mismos que tienen como premisa la confidencialidad de los datos personales de los participantes, así como la información a detalle obtenida a través de los cuestionarios, los cuales fueron para uso exclusivo de los investigadores y con fines académicos.

\section{Resultados}

De acuerdo con los resultados obtenidos de la aplicación del instrumento de recolección de información, se observa lo siguiente: 


\section{Revista Iberoamericana \\ de las Ciencias Sociales y \\ Humanísticas}

\section{D0. Información general}

Tabla 1. Cuestionamientos y respuestas relativos a la información general

\begin{tabular}{|l|}
\hline Sexo \\
\hline 451 (hombres), 549 (mujeres) \\
\hline Edad \\
\hline 317 (18-30 años), 474 (31-50 años), 209 (51 o más ) \\
\hline ¿Tiene conexión de potable? \\
\hline 968 (sí), 32 (no) \\
\hline Escolaridad \\
\hline 18 (ninguna), 137 (primaria), 260 (secundaria), 337 (bachillerato), 248 (universidad) \\
\hline ¿Cuántas personas habitan su domicilio? \\
\hline 504 (1-3), 460 (4-6), 36 (más de 6) \\
\hline ¿Cuenta con jardín en su domicilio? \\
\hline 476 (sí), 524 (no) \\
\hline ¿Cuántos baños tiene su domicilio? \\
\hline 320 (1), 474 (2), 181 (3 o más), 25 (letrina) \\
\hline
\end{tabular}

\section{Fuente: Elaboración propia}

En la Tabla 1, observamos que la mayoría de la población encuestada fueron mujeres. La edad con mayor categoría se encuentra entre los 31 a 50 años de edad. Casi la totalidad cuenta con conexión de agua potable y la escolaridad más alta fue bachillerato. Con respecto de las personas que habitan por domicilio, encontramos que las categorías más altas se encuentran entre 1-3 y 4-6. Asimismo, la mayoría no tiene un jardín dentro de su domicilio y el número de baños en el domicilio que presentó las categorías más altas fue de dos y uno por domicilio; sin embargo, 181 personas tiene 3 o más y 25 cuentan con letrina. Lo anterior permite señalar, en la presente dimensión, como dato relevante, que hay un alto índice de habitantes por domicilio, lo que representa mayor cantidad de agua utilizada para satisfacer las necesidades del servicio, aunado a que el agua utilizada para mantener los jardines se eleva al ser casi la mitad de la población quien sí tiene uno. Por último, con respecto de los baños en el domicilio, la sumatoria de quienes están en la categoría de dos y tres o más, es mayor que las personas que solamente cuentan con un baño de servicio. Además, todavía hay población que utiliza letrinas para satisfacer esa necesidad, lo que representa un aspecto para considerar como parte de la mejora en los servicios que provee el organismo. 


\section{Revista Iberoamericana de las Ciencias Sociales y Humanísticas}

\section{D1. Visibilidad de la problemática del agua}

Tabla 2. Cuestionamientos y respuestas relativos a la visibilidad de la problemática del agua

¿EI agua que pueden consumir las personas es un recurso que se puede acabar?

913 (sí), 87 (no)

¿Si seguimos como ahora, nuestros hijos o descendientes no tendrán agua para consumir en el futuro?

818 (sí), 182 (no)

¿Las personas que tienen servicio de agua potable en sus casas, la desperdician?

862 (sí), 138 (no)

¿El calentamiento global está reduciendo las fuentes de agua en el mundo?

870 (sí), 130 (no)

¿Actualmente hay problemas de escasez de agua potable en el mundo?

894 (sí), 106 (no)

¿Todas las personas en el país tienen acceso al agua potable?

620 (sí), 380 (no)

¿Si las persona utilizaran mejor el agua que llega a sus hogares, más personas tendrían acceso al agua potable?

863 (sí), 137 (no)

¿Hay escasez de agua en Xalisco?

786 (sí), 214 (no)

¿La problemática del agua es una de sus preocupaciones para el futuro inmediato?

817 (sí), 183 (no)

¿El agua con que se cuenta para abastecer al municipio de Xalisco se puede acabar?

841 (sí), 159 (no)

¿Conoce de dónde proviene el agua que llega a su domicilio?

571 (sí), 429 (no)

¿Conoce de qué manera llega el agua a su domicilio?

659 (sí), 341 (no)

¿Conoce en qué condiciones y calidad llega el agua a su domicilio?

620 (sí), 380 (no)

¿Cuál es el principal problema que enfrenta el mundo actualmente con respecto del medio ambiente?

716 (contaminación de ríos/mares), 284 (la falta de agua)

Fuente: Elaboración propia

Con respecto de la visibilidad de la problemática del agua, en la Tabla 2 se puede observar que la población expresa claramente que están conscientes de aquellas situaciones que ponen en riesgo el acceso al vital líquido; señalan, además, que causas como el cambio climático influyen en la disminución de acceso al recurso; también, existe una consideración para la escasez del agua 


\section{Revista Iberoamericana \\ de las Ciencias Sociales y \\ Humanísticas}

ISSN: $2395-7972$

en el municipio para un futuro inmediato, reconociendo que el acceso al recurso hídrico puede ser finito. Aunado a lo anterior, se observa que tienen conocimientos de dónde, cómo y las condiciones de cómo obtienen el suministro de agua en sus domicilios. Por último, consideran que la contaminación del agua en ríos y mares es una problemática que impacta en el medio ambiente.

Tabla 3. El resto de cuestionamientos y respuestas relativos a la visibilidad de la problemática del agua

\begin{tabular}{|l|}
\hline ¿Por qué o para qué considera que el agua es importante? \\
\hline Considero que los seres vivos necesitamos agua para vivir (709), Es lo principal, sin agua \\
no hay nada (423), Para la salud e higiene (383), Es necesaria para bañarse y lavar cosas \\
(300), Es necesaria para cualquier población (297), Porque es vital para las personas (290), \\
Para beber y cocinar (258), Porque en el cuerpo tenemos $70 \%$ del agua y la necesitamos \\
(220), Porque es una necesidad para nuestros hijos (208), Porque sin agua habría sequía en \\
el mundo (124) \\
¿Para qué usa con mayor frecuencia el agua? \\
Aseo personal (806), Lavar ropa (568), Limpiar el hogar (542), Lavar trastes (534), Baño \\
(522), Cocinar (480), Lavar alimentos (424), Regar las plantas (302), Lavar transporte \\
(193) \\
Si en un futuro inmediato hubiera una emergencia y el agua escasea ¿quiénes serían \\
los más afectados? \\
Toda la población (846), Las personas de recurso bajos (187), Enfermos y hospitales (112), \\
Ancianos (90), Las personas con recursos medios (72), Niños (71), Empresas (63), \\
Comercios (62), Las personas con recursos altos (43), Nadie (36) \\
\hline
\end{tabular}

Fuente: Elaboración propia

En la misma dimensión también se destaca en la Tabla 3, que las respuestas con mayor frecuencia se encuentran en el reconocimiento de la necesidad del vital líquido, considerando además la importancia del agua para cualquier actividad, incluyendo la salud e higiene y actividades domésticas, seguido de considerarla una necesidad para la familia y las repercusiones por sequía que puede representar el que no se cuente con el recurso hídrico. Además de expresar la mayor frecuencia para el uso del agua en actividades domésticas cotidianas; el aseo personal es la que registra el mayor número. Con respecto de quiénes se verían afectados en caso de alguna emergencia para la obtención del agua, expresan mayormente que toda la población, indistintamente de que algunas otras señalan grupos específicos de personas tales como personas de recursos bajos, enfermos, hospitales y ancianos. Un dato importante es que 36 personas consideran que nadie se afectaría en caso de que dicha situación existiese. 


\section{Revista Iberoamericana \\ de las Ciencias Sociales y \\ Humanísticas}

\section{D2. Servicios}

Tabla 4. Cuestionamientos y respuestas relativos a los servicios de agua

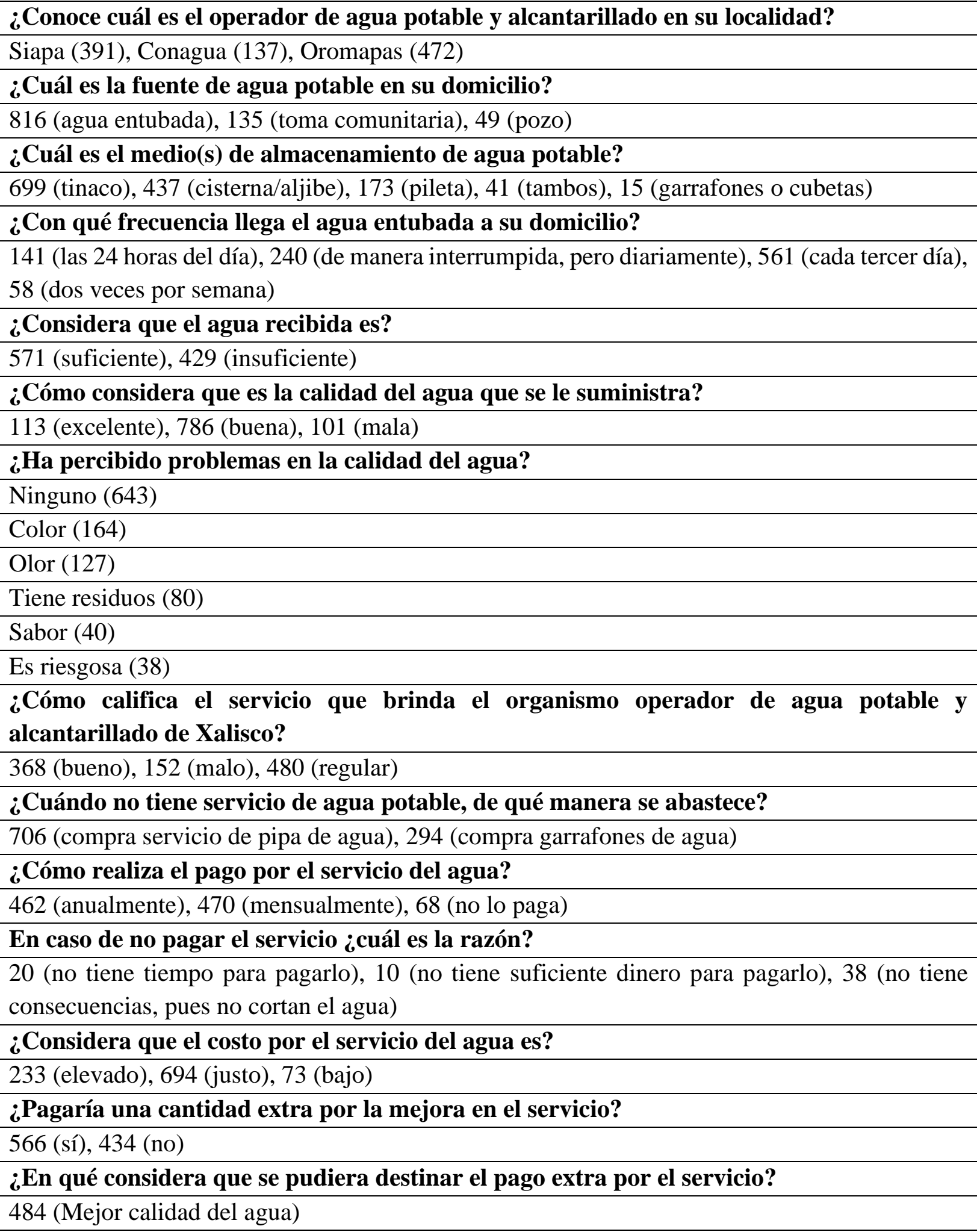


Revista Iberoamericana

de las Ciencias Sociales y

Humanísticas

ISSN: $2395-7972$

463 (Reparar fugas en la calle con mayor rapidez)

220 (Mejor continuidad y presión del agua)

176 (Mejora de las oficinas para atención)

¿Cuál considera que es el/los problemas más constantes en la prestación del servicio?

198 (atención al cliente), 484 (atención a fugas de agua), 455 (abastecimiento de agua), 151 (calidad del agua)

¿Ha presentado en el último año alguna reclamación en relación con el suministro del agua por alguna de las siguientes causas?

173 (problemas en el cobro), 286 (corte de suministro), 348 (condiciones en el servicio), 344 (fugas no atendidas)

¿Considera que si tuviera un medidor de consumo de agua, utilizaría el recurso de mejor manera?

882 (sí), 118 (no)

Fuente: Elaboración propia

La presente dimensión (véase Tabla 4) permitió descubrir que hay altos niveles de desconocimiento acerca de la información relacionada con el organismo operador. Asimismo, la fuente de agua potable señalada con mayor frecuencia es la del agua entubada y de la menor, a través de pozos. Otro de los aspectos importantes es que aunque la mayoría cuenta con tinacos como medio de almacenamiento, también hay personas que utilizan garrafones o cubetas por la falta de medios.

En relación con el suministro del servicio, la mayoría recibe el líquido cada tercer día, considerando que es de manera suficiente y con buena calidad, aunque se observan datos que permiten determinar la presencia de aspectos que, sin duda, manifiestan problemas en la calidad del agua, tales como olor, color, sabor y residuos, por tanto, pueden existir riesgos en el uso.

Referente a la percepción sobre el servicio que ofrece el organismo operador, se aprecia que la mayoría de la población lo encuentra regular. Y se puede establecer, a partir de esta consideración, el origen de la problemática que la población tiene que enfrentar cuando existe un desabasto del recurso, lo que conlleva a realizar una inversión adicional para la compra de pipas de agua o garrafones de agua para satisfacer las necesidades. En consecuencia, se infiere que lo anterior impacta directamente en la cultura del pago, debido a que, aunque la mayoría paga anualmente, hay 62 personas que no realizan pago alguno por el servicio a razón de no tener consecuencias. Dentro de la situación planteada, sin embargo, sobresale un punto favorable: la población considera que el costo es justo y estarían dispuestos a pagar más para la mejora del servicio, específicamente señalan que este recurso se utilice en mejorar la calidad del agua, reparar fugas y mejorar la continuidad y presión para el suministro. 


\section{Revista Iberoamericana \\ de las Ciencias Sociales y \\ Humanísticas}

ISSN: $2395-7972$

Dentro de las problemáticas referidas al servicio, las respuestas con mayor frecuencia señalan la atención a fugas y el abastecimiento del recurso hídrico, situaciones que tienen mayor injerencia para presentar reclamaciones al organismo operador de agua potable y alcantarillado. Un aspecto importante es la disponibilidad que expresan sobre la posibilidad de implementar medidores de consumo de agua para una adecuada medición del uso y cobro del servicio.

\section{D3. Cuidado y conservación}

Tabla 5. Cuestionamientos y respuestas relativos al cuidado y conservación del recurso

\begin{tabular}{|l|}
\hline ¿Cuál o cuáles considera que son las razones por las que no cuida el agua? \\
\hline 407 (desinformación) \\
\hline 390 (no me doy cuenta cuando la desperdicio) \\
\hline 238 (desinterés) \\
\hline 152 (el servicio es económico y no hay sanciones si la desperdicio) \\
\hline 133 (otras prioridades) \\
\hline 50 (no sé) \\
\hline 48 (no creo que el agua se acabe) \\
\hline ¿Cuál de las siguientes acciones realiza para cuidar el agua? \\
\hline 539 (cerrar adecuadamente las llaves para que no se desperdicie) \\
\hline 420 (no usar agua de más) \\
\hline 403 (minimizar su uso) \\
\hline 304 (enseñar a la familia sobre el cuidado del agua) \\
\hline 238 (no durar mucho tiempo bañándome) \\
\hline 213 (cuidar el estado de las llaves o tuberías para no desperdiciar) \\
\hline 122 (reutilizar el agua) \\
\hline 79 (utilizar agua de lluvia) \\
\hline ¿Conoce algún método para reutilizar el agua? \\
\hline 525 (sí), 475 (no) \\
\hline ¿Reutiliza el agua? \\
\hline 361 (sí), 639 (no) \\
\hline ¿Cuál o cuáles acciones realiza para conservar limpia el agua almacenada? \\
\hline 642 (lavar por lo menos una vez al año el tinaco) \\
\hline 296 (lavar el tinaco en un periodo mayor a un año) \\
\hline 201 (lavar por lo menos una vez al año el aljibe) \\
\hline 109 (lavar el aljibe en un periodo mayor a un año) \\
\hline 86 (lavar por lo menos una vez al año los depósitos del inodoro) \\
\hline \\
\hline
\end{tabular}




\section{Revista Iberoamericana \\ de las Ciencias Sociales y \\ Humanísticas}

ISSN: $2395-7972$

\begin{tabular}{|l|}
\hline 37 (lavar los tambos o cubetas de almacenamiento cuando se vacían) \\
\hline ¿El nivel de responsabilidad que tiene para cuidar el agua es? \\
\hline 223 (máximo), 548 (medio), 200 (poco), 29 (nulo) \\
\hline ¿Cuáles son los motivos por los que considera que se desperdicia el agua? \\
\hline 567 (no hay conciencia en la población o por ignorancia) \\
\hline 508 (mal uso) \\
\hline 323 (poca importancia para cuidar el agua) \\
\hline 216 (falta de campañas para concienciar sobre la importancia del agua) \\
\hline 214 (se usa el agua para cualquier actividad) \\
\hline 174 (ausencia de regulación para el uso del agua) \\
\hline 157 (mucho uso para esparcimiento o diversión) \\
\hline 150 (el agua es barata) \\
\hline 104 (infraestructura en mal estado) \\
\hline 69 (no sé cómo cuidarla) \\
\hline 30 (el agua abunda y es inagotable) \\
\hline 15 (no sé) \\
\hline ¿Quién considera que es principal responsable de cuidar el agua? \\
\hline 497 (la población en general) \\
\hline 307 (todos los anteriores) \\
\hline 174 (el gobierno y los políticos) \\
\hline 149 (los usuarios) \\
\hline 83 (el organismo que otorga el servicio) \\
\hline
\end{tabular}

Fuente: Elaboración propia

En la Tabla 5, es posible observar que la falta de información es uno de los aspectos sustanciales para que la población no cuide el recurso hídrico, aunado a que todavía no son conscientes de cuándo están desperdiciando el líquido; otro de los aspectos es el desinterés. Es oportuno señalar que, aunque en menor medida, también señalan el hecho de que, al no existir sanciones reales para quienes incurran en el desperdicio o mal uso, aprovechan esta circunstancia para desperdiciarla. Por otro lado, con respecto del cuidado, la mayoría de las frecuencias indican que están pendientes de realizar actividades para no desperdiciarla y cuidarla, y una pequeña parte de la población hace uso de métodos para el uso del agua proveniente de otras fuentes como la lluvia. Además, en relación con la reutilización del recurso, aunque manifiestan conocer métodos, estos no son utilizados. Otro aspecto importante son las acciones para mantener los medios de almacenamiento en condiciones adecuadas, donde las frecuencias indican que por lo menos una vez al año dan mantenimiento a los medios de almacenamiento como tinacos, aljibes, inodoro o cubetas. 


\section{Revista Iberoamericana \\ de las Ciencias Sociales y \\ Humanísticas}

ISSN: $2395-7972$

En el caso de la responsabilidad que asumen frente al cuidado del agua, un poco más de la mitad de la población estableció un nivel medio de responsabilidad, seguido de uno máximo y poco; sin embargo, 29 personas señalan que su nivel es nulo.

Con respecto de los motivos para desperdiciarla, las mayores frecuencias señalan que no existe una conciencia para el cuidado y la ignorancia acerca de las maneras para cuidarla. Por tanto, existe un mal uso y poca importancia para cuidar el vital líquido. Asimismo, la falta de campañas, ausencia de regulación, el uso para cualquier actividad, el bajo costo, infraestructura en mal estado y el pensar que es inagotable son causas para desperdiciarla. Por último, consideran que el principal responsable del cuidado es la misma población, seguido del Gobierno y los políticos, los usuarios y el organismo en conjunto.

\section{D4. Conocimiento y participación}

Tabla 6. Cuestionamientos y respuestas relativos al conocimiento y participación



¿Conoce el portal de información de OROMAPAS?

239 (sí), 761 (no)

¿Considera que es necesario que el gobierno municipal implemente campañas de información para cuidado del agua?

814 (sí), 186 (no)

¿Ha consultado información acerca de la situación de la problemática del agua en el mundo?

381 (sí), 619 (no)

¿Conoce el alto riesgo en que se encuentra nuestro país en los próximos años para abastecer de agua a la población?

532 (sí), 468 (no)

¿Estaría dispuesto(a) a participar en pláticas o actividades para informarse acerca de los temas relacionados con el agua?

770 (sí), 230 (no)

Fuente: Elaboración propia

En la dimensión sobre conocimiento y participación (véase Tabla 6), resalta que no cuentan con información suficiente para realizar actividades que les permitan cuidar o preservar el agua. De igual manera, desconocen los medios oficiales para obtener información del organismo operador, y señalan, además, la importancia de que el municipio implemente campañas 


\section{Revista Iberoamericana \\ de las Ciencias Sociales y \\ Humanísticas}

ISSN: 2395 - 7972

informativas. Otro aspecto a resaltar es que en un contexto local tienen conocimiento de la problemática del recurso hídrico, por tanto, son conscientes del riesgo en nuestro país para el abastecimiento de agua; sin embargo, en un contexto global existe un desconocimiento de la temática.

\section{D5. Marco legal}

Tabla 7. Cuestionamientos y respuestas relativos al marco legal

¿Conoce las leyes relacionadas con el agua para nuestro estado?

179 (sí), 821 (no)

¿Conoce las leyes relacionadas con el agua para el municipio de Xalisco?

132 (sí), 868 (no)

¿Ha consultado alguna vez las leyes existentes en materia de agua para nuestro estado o municipio?

187 (sí), 813 (no)

¿Considera importante que existan leyes, normas o reglamentos que regulen de forma estricta el consumo de agua?

755 (sí), 245 (no)

¿Considera importante que las autoridades cumplan las leyes existentes en materia de agua potable y alcantarillado?

868 (sí), 132 (no)

¿Considera importante que los usuarios cumplan las leyes existentes en materia de agua potable y alcantarillado?

889 (sí), 111 (no)

Fuente: Elaboración propia

Finalmente, en la Tabla 7 se observa con respecto a la información relacionada con el marco legal, un nivel muy alto de falta de información o consulta sobre la existencia de regulaciones. No obstante, es importante para la población que exista, así como que la autoridad dé cumplimiento en su aplicación. Asimismo, que el usuario también cumpla con aquello estipulado en el marco regulatorio. 


\section{Revista Iberoamericana \\ de las Ciencias Sociales y \\ Humanísticas}

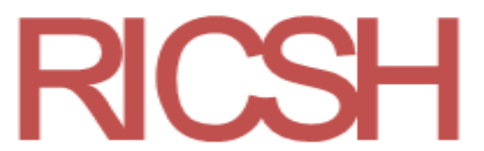

ISSN: $2395-7972$

\section{Discusión}

Debido a que el término analfabetismo hídrico no es de uso común en los estudios actuales, las referencias remiten a la educación ambiental. El espectro de esta, sin embargo, abarca un sinfín de problemáticas. Como se ha visto, el presente proyecto se circunscribe al recurso hídrico, y los resultados indican que la población no está informada respecto de aquellos aspectos que se relacionan con el agua. Por tanto, esto limita la actuación de la población y el propio Gobierno a través del organismo operador de agua potable, alcantarillado y saneamiento del municipio de Xalisco, Nayarit, para el uso, cuidado y conservación del recurso, además de la posible implementación de un modelo de gobernanza que satisfaga las necesidades de la demanda. Se sugiere que en estudios futuros se consideren las 43 localidades, a efectos de tener un panorama completo de la problemática en el municipio.

De acuerdo con el Programa Hidrológico Internacional de la Unesco, el presente estudio contribuye a nivel local en la identificación de posibles rutas de acción para el cumplimiento de los objetivos específicos que señalan la importancia de desarrollar nuevas estrategias de educación hídrica en las comunidades (Organización de las Naciones Unidas para la Educación, la Ciencia y la Cultura [Unesco], 2016). Por otra parte, una aproximación al presente estudio la encontramos en la tesis de maestría intitulada Hacia una cultura sustentable del agua en la población adulta del municipio de Naolinco, Veracruz, desarrolla por Araceli Mora Castillo (2016), donde sobresale que la mayoría de la población allí encuestada (una muestra conformada por 416 personas) no se considera responsable de encontrar o colaborar en la solución. También los encuestados manifiestan que aquellos que participan por considerarse responsables piensan que dicha participación no influirá en un cambio sustancial. En este sentido, encontramos una diferencia sustancial en los hallazgos de nuestro proyecto, donde la población sí reconoce su responsabilidad y considera su participación en actividades informativas o campañas para el cuidado y conservación del recurso hídrico. Por otra parte, existe una coincidencia en la consideración de la educación para promover un cambio en actitudes, valores, creencias y modos de vida para dirigirnos hacia una cultura del agua (Mora, 2016).

Otro estudio publicado en la Revista Mexicana de Opinión Pública de Márquez y Ortega (2017), "Percepción social del servicio de agua potable en el municipio de Xalapa, Veracruz", desarrolló una encuesta que fue aplicada a 280 personas de la comunidad xalapeña, y presenta entre los resultados la aceptación mayoritaria del organismo que provee el servicio, aunque se identificaron áreas del servicio como vulnerables, por lo que se propone elaborar acciones 


\section{Revista Iberoamericana \\ de las Ciencias Sociales y \\ Humanísticas}

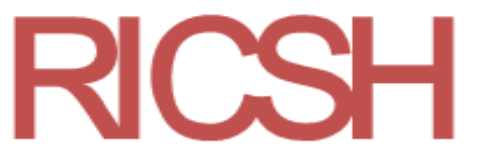

ISSN: $2395-7972$

estratégicas para una mayor calidad en el servicio. En relación con los resultados de nuestra investigación, encontramos semejanzas en la percepción de la ciudadanía con respecto de las variables analizadas en el estudio señalado. Sin embargo, la diferencia de variables no permite establecer un análisis más allá de cómo visualizan el servicio otorgado por el organismo (Márquez y Ortega, 2017).

Con respecto del acceso al agua, los autor del proyecto intitulado "Acceso al agua para uso doméstico de caso en Berriozabal, Chiapas”, publicado en 2016 en la Revista Mexicana de Ciencias Agrícolas, encuestaron a 1099 grupos domésticos, y señalan entre los resultados que el acceso al agua en la zona rural es mayor que en las áreas urbanas, donde deben comprar pipas o extraer de pozos para cubrir las necesidades domésticas. Un dato relevante es que el agua proviene de manantiales, por lo cual, las zonas rurales tienen acceso al recurso hídrico. En referencia con nuestra investigación, el suministro de agua en su mayoría es a través de infraestructura del organismo operador, sin embargo, también hay una parte de la población que la extrae de pozos y, en la mayoría de los casos, cuando existe desabasto requieren comprar pipas de agua o garrafones para satisfacer la demanda (Gutiérrez, Ayala, Zapata, Salvatierra y Nazar, 2016).

Finalmente, es oportuno mencionar que, de acuerdo con los resultados, la corresponsabilidad se convierte en un aspecto fundamental para el cuidado y preservación de los recursos hídricos. Sin embargo, la participación de la sociedad requiere de una acción llevada a cabo en conjunto con el Gobierno municipal a través de políticas públicas instituidas y basadas en la gobernabilidad y gobernanza del agua (Comisión Nacional del Agua [Conagua], 2014). Este reto puede ser enfrentado a partir la disminución del analfabetismo hídrico y del involucramiento de todos los actores relacionados con la problemática del agua, tales como Gobierno, sector privado y la comunidad. Con lo anterior se podrá impactar en las políticas que tienen repercusiones en el desarrollo humano; empoderar a las comunidades y a los ciudadanos; acordar colectivamente tanto los problemas comunes como la forma en que estos deberán ser atendidos; medir y evaluar los impactos que las políticas públicas tienen en el desarrollo humano, además de propiciar la participación activa para la conservación del recurso hídrico. 


\section{Revista Iberoamericana \\ de las Ciencias Sociales y \\ Humanísticas}

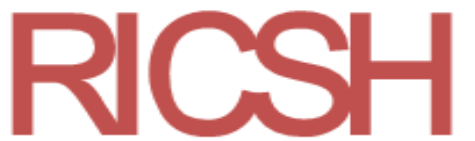

ISSN: $2395-7972$

\section{Conclusiones}

El analfabetismo hídrico es una realidad presente en la población encuestada del municipio de Xalisco. Sin duda las características relacionadas a las condiciones de la vivienda representan un aspecto sustancial para considerar posibles escenarios de acción con respecto a cómo solucionar la demanda del recurso hídrico. Aunado a esta consideración, es evidente la falta de información relacionada con los servicios, cuidado y conservación, además de métodos alternativos para la obtención del agua. Aunque los resultados señalan que la población tiene información sobre la problemática del agua, misma que puede poner en riesgo el acceso al vital líquido, es necesario fortalecer el acceso a la información a toda la población. Esto con la finalidad de impactar sustancialmente en la percepción sobre la realidad que existe en el municipio, el país y el mundo sobre el acceso al agua.

Un punto relevante es también la poca información sobre el organismo operador que brinda el servicio a la población, y que todavía hay parte de la población que se abastece a partir de pozos. Indudablemente, se observa que los medios utilizados para el almacenamiento pueden ser considerados adecuados. Sin embargo, habrá que establecer criterios de atención para aquella población que actualmente utiliza medios no adecuados como garrafones o cubetas. Aunado a lo anterior, el suministro presenta deficiencias que generan inconformidad en la población, por lo tanto, esto tiene un impacto directo en la cultura del pago, que afecta sustancialmente los ingresos del organismo, y, al no aplicar la regulación existente de manera tal que la población sea sujeto de las consecuencias, esta continuará evadiendo la responsabilidad que conlleva la prestación del servicio. Sin embargo, encontramos aceptación para la implementación de medidores de consumo de agua, lo que propiciaría una adecuada medición del recurso utilizado y, por ende, el cobro de manera más objetiva.

Además, la falta de información en la población genera una actitud que redunda en la falta de cuidado y conservación del agua, por tanto, se requiere la implementación de campañas que permitan asumir la responsabilidad correspondiente y que estas fomenten la participación ciudadana. También es importante señalar que la información deberá ser accesible para todo tipo de población, es decir, desde medios tradicionales de comunicación hasta medios digitales; el propósito principal es el de proporcionarles un panorama desde los contextos internacionales, nacionales, regionales y locales sobre la problemática relacionada con el agua, la legislación existente y las sanciones correspondientes. Lo anterior a efectos de que pueda incidir en la forma en que la perciben, además de propiciar la generación de una participación más activa y adecuada 


\section{Revista Iberoamericana \\ de las Ciencias Sociales y \\ Humanísticas}

ISSN: $2395-7972$

a las necesidades para fortalecer la cultura sobre el agua en la población. Finalmente, lo que se requiere es una decisión responsable para realizar cambios necesarios. El analfabetismo hídrico nos obliga a impulsar una educación para el cuidado y una nueva cultura del agua. Lo anterior basado en una nueva escala de valores, percepción de la naturaleza, principios éticos, estilos de vida y un cambio cultural propiciado por los responsables de la gestión de los recursos hídricos.

\section{Referencias}

Comisión Nacional del Agua [Conagua]. (2013). Estadística del Agua en México. México: Secretaría de Medio Ambiente y Recursos Naturales.

Comisión Nacional del Agua [Conagua ]. (2014). Programa Nacional Hídrico 2014-2018. México: Secretaría del Medio Ambiente y Recursos Naturales.

Domínguez, J. (2013). Obstáculos para una gobernanza efectiva del agua en México. Estudio de la región hidrológica X del Golfo Centro. Oswald, Ú. (Coord.a), Retos de la legislación del agua en México (pp. 625-638). Cuernavaca, México: UNAM/Centro Regional de Investigaciones Multidisciplinarias.

Gutiérrez, V., Ayala, M., Zapata, E., Salvatierra, B. y Nazar, A. (2016). Acceso al agua para uso doméstico estudio de caso en Berriozábal, Chiapas. Revista Mexicana de Ciencias Agrícolas, (17), 3596-3605.

Jiménez, B. (2015). Seguridad Hídrica: Retos y respuestas, la fase VIII del Programa Hidrológico Internacional de la UNESCO (2014-2021). Aqua-LAC, 7(1), 20-27.

Márquez, O. y Ortega, M. (2017). Percepción social del servicio de agua potable en el municipio de Xalapa, Veracruz. Revista Mexicana de Opinión Publica, 23, 41-59. Recuperado de https://www.sciencedirect.com/science/article/pii/S1870730017300030.

Mora, A. (2016). Hacia una cultura sustentable del agua en la población adulta del municipio de Naolinco, Veracruz. (tesis de maestría). Universidad Veracruzana, Veracruz, Recuperado de https://cdigital.uv.mx/handle/123456789/42120?show=full.

Musetta, P. (2009). Participación y gobernanza. El modelo de gobierno del agua en México. Espacios Públicos, 12(25), 66-84.

Organización de las Naciones Unidas para la Educación, la Ciencia y la Cultura [Unesco]. (17 de junio de 2016). Educación y Cultura del Agua: clave para la seguridad hídrica. Organización de las Naciones Unidas para la Educación, la Ciencia y la Cultura. 


\section{Revista Iberoamericana \\ de las Ciencias Sociales y \\ Humanísticas}

Recuperado

de

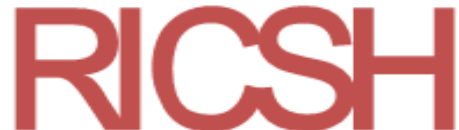

ISSN: $2395-7972$

https://es.unesco.org/themes/garantizar-suministroagua/hidrologia/educacion/informal.

Organización de las Naciones Unidas para la Educación, la Ciencia y la Cultura [Unesco]. (13 de octubre de 2018). UNESDOC Biblioteca Digital: Reseña. Agua para todos, Agua para la vida. Organización de las Naciones Unidas para la Educación, la Ciencia y la Cultura. Recuperado de https://unesdoc.unesco.org/ark:/48223/pf0000129556_spa.

Organización de los Estados Americanos [OEA]. (2017). Hacia una buena Gobernanza para la Gestión Integrada de los Recursos Hídricos. Recuperado de http://www.oas.org/en/sedi/dsd/iwrm/past\%20events/D7/6\%20WWF-

GOBERNANZA\%20Final.pdf.

Organización para la Cooperación y el Desarrollo Económicos [OCDE]. (2012). Gobernabilidad del Agua en América Latina y El Caribe: Un enfoque multinivel. París, Francia: Editions OCDE.

Programa de las Naciones Unidas para el Desarrollo [PNUD]. (25 de enero de 2016). Objetivo 6. Agua limpia y saneamiento. Programa de las Naciones Unidas para el Desarrollo. México. Recuperado de http://www.mx.undp.org/content/mexico/es/home/post-2015/sdgoverview/goal-6.html.

Rosas, F., Campos, H. y Calderon Maya, J. (2012). Elementos conceptuales para el análisis de la gobernanza territorial. Quivera. Revista de Estudios Territoriales, 14(2), 113-135. 
Revista Iberoamericana

de las Ciencias Sociales y

Humanísticas

ISSN: $2395-7972$

\begin{tabular}{|c|c|}
\hline Rol de Contribución & Autor (es) \\
\hline Conceptualización & $\begin{array}{l}\text { Nicté Rosas Topete (igual), Edgar Gabriel Ávila Verdín } \\
\text { (igual), Isma Sandoval Galaviz (igual) }\end{array}$ \\
\hline Metodología & $\begin{array}{l}\text { Nicté Rosas Topete (igual), Edgar Gabriel Ávila Verdín } \\
\text { (igual), Isma Sandoval Galaviz (apoyo) }\end{array}$ \\
\hline Software & $\begin{array}{l}\text { Nicté Rosas Topete (principal), Edgar Gabriel Ávila Verdín } \\
\text { (apoyo), Isma Sandoval Galaviz (apoyo) }\end{array}$ \\
\hline Validación & $\begin{array}{l}\text { Nicté Rosas Topete (principal), Edgar Gabriel Ávila Verdín } \\
\text { (apoyo), Isma Sandoval Galaviz (apoyo) }\end{array}$ \\
\hline Análisis Formal & $\begin{array}{l}\text { Nicté Rosas Topete (igual), Edgar Gabriel Ávila Verdín } \\
\text { (igual), Isma Sandoval Galaviz (igual) }\end{array}$ \\
\hline Investigación & $\begin{array}{l}\text { Nicté Rosas Topete (igual), Edgar Gabriel Ávila Verdín } \\
\text { (igual), Isma Sandoval Galaviz (igual) }\end{array}$ \\
\hline Recursos & $\begin{array}{l}\text { Nicté Rosas Topete (principal), Edgar Gabriel Ávila Verdín } \\
\text { (apoyo), Isma Sandoval Galaviz (apoyo) }\end{array}$ \\
\hline Curación de datos & $\begin{array}{l}\text { Nicté Rosas Topete (principal), Edgar Gabriel Ávila Verdín } \\
\text { (apoyo), Isma Sandoval Galaviz (apoyo) }\end{array}$ \\
\hline $\begin{array}{l}\text { Escritura - Preparación del } \\
\text { borrador original }\end{array}$ & $\begin{array}{l}\text { Nicté Rosas Topete (principal), Edgar Gabriel Ávila Verdín } \\
\text { (apoyo), Isma Sandoval Galaviz (apoyo) }\end{array}$ \\
\hline $\begin{array}{l}\text { Escritura - Revisión y } \\
\text { edición }\end{array}$ & $\begin{array}{l}\text { Nicté Rosas Topete (principal), Edgar Gabriel Ávila Verdín } \\
\text { (apoyo), Isma Sandoval Galaviz (apoyo) }\end{array}$ \\
\hline Visualización & $\begin{array}{l}\text { Nicté Rosas Topete (principal), Edgar Gabriel Ávila Verdín } \\
\text { (apoyo), Isma Sandoval Galaviz (apoyo) }\end{array}$ \\
\hline Supervisión & $\begin{array}{l}\text { Nicté Rosas Topete (principal), Edgar Gabriel Ávila Verdín } \\
\text { (apoyo), Isma Sandoval Galaviz (apoyo) }\end{array}$ \\
\hline $\begin{array}{l}\text { Administración de } \\
\text { Proyectos }\end{array}$ & $\begin{array}{l}\text { Nicté Rosas Topete (principal), Edgar Gabriel Ávila Verdín } \\
\text { (apoyo), Isma Sandoval Galaviz (apoyo) }\end{array}$ \\
\hline Adquisición de fondos & $\begin{array}{l}\text { Nicté Rosas Topete (principal), Edgar Gabriel Ávila Verdín } \\
\text { (apoyo), Isma Sandoval Galaviz (apoyo) }\end{array}$ \\
\hline
\end{tabular}

